\title{
The Influence of the Magnitude of Gravitational Acceleration on the Marangoni Convection About an Isolated Bubble under a Heated Wall
}

Séamus M. O’shaughnessy and Anthony J. Robinson

\section{QUERY SHEET}

This page lists questions we have about your paper. The numbers displayed at left can be found in the text of the paper for reference. In addition, please review your paper as a whole for correctness.

Q1: Au: Update available? 


\title{
The Influence of the Magnitude of Gravitational Acceleration on the Marangoni Convection About an Isolated Bubble under a Heated Wall
}

\author{
5 SÉAMUS M. O'SHAUGHNESSY and ANTHONY J. ROBINSON
}

Department of Mechanical \& Manufacturing Engineering, Trinity College Dublin, Dublin, Ireland

\begin{abstract}
Thermocapillary or Marangoni convection is the liquid motion caused by surface tension variation in the presence of a temperature gradient along a gas-liquid or vapor-liquid interface. This work numerically investigates the effect of the magnitude of gravitational acceleration on the flow and temperature fields resulting from the presence of a hemispherical air bubble of constant radius of $1.0 \mathrm{~mm}$, situated on a heated wall immersed in a liquid silicone oil layer of constant depth of $5.0 \mathrm{~mm}$. The model is oriented such that the Marangoni and gravitational forces act to oppose one another. To elucidate the effect of gravity on Marangoni flow and heat transfer, the simulations were carried out for a silicone oil of Prandtl number 83, at a Marangoni number of 915. The gravity levels tested were $0 \mathrm{~g}, 0.01 \mathrm{~g}, 0.1 \mathrm{~g}, 0.25 \mathrm{~g}, 0.5 \mathrm{~g}, 0.75 \mathrm{~g}$, and $1 \mathrm{~g}$, where $\mathrm{g}$ represents the earth gravitational acceleration of $9.81 \mathrm{~m} / \mathrm{s}$. The influence of the magnitude of gravitational acceleration on the velocity profile along the bubble interface and on the location of maximum velocity was analyzed. It was found that the gravity level affects the velocity profile by influencing the interfacial temperature gradient, but that the location of maximum velocity was almost independent of gravity level. The increase in heat flux on the wall to which the bubble is attached was calculated and it has been determined that local heat transfer enhancement of up to nearly 1.7 times that of the conduction only case can be achieved for the parameter range tested. Furthermore, local enhancement was observed to occur up to a distance of seven bubble radii for the zero-gravity case, but increased gravity levels cause a reduction in the effective radius of enhancement. The influence of the Marangoni flow on the heat transfer for the opposite wall has also been analyzed.
\end{abstract}

\section{INTRODUCTION}

Thermocapillary or Marangoni convection is the liquid motion brought about by surface tension variation in the presence of a temperature gradient along a gas-liquid or vapor-liquid interface. The attractive forces between the liquid molecules at the interface cause surface tension effects [1]. When a small gas bubble is situated on a heated wall, the difference in temperature between the overheated base and the cooler tip of the bubble causes the surface tension gradient [2]. The net surface tension force on any element of the interface is opposed by viscous shear stresses in both the gas and liquid phases [2], but since the shear

This work was part supported by the Irish Research Council for Science, Engineering and Technology (IRCSET) Embark Initiative.

Address correspondence to Dr. Anthony J. Robinson, Department of Mechanical \& Manufacturing Engineering, Parsons Building, Trinity College Dublin, Dublin 2, Ireland. E-mail: arobins@tcd.ie stress in the liquid phase is typically much greater than that of the gas phase, the gas phase may be neglected, as has been the case in previous numerical investigations [2,3]. Most natural convection processes on earth are buoyancy driven, caused by the density gradients within fluids resulting from temperature gradients within the system. Marangoni convection, however, is independent of gravitational forces, and has therefore been suggested by some authors as a possible solution to thermal management problems in space [1].

The majority of fluids exhibit a negative temperature derivative of surface tension. As the temperature is increased, the surface tension decreases. In most fluids, this will result in a flow of fluid from warm regions to cold regions. A typical scenario for bubble-induced thermocapillary convection is shown in Figure 1. An air bubble is situated under a heated wall, immersed in a cooler liquid layer. The fluid close to the triple contact point is accelerated along the liquid-vapor interface. This results in a flow of liquid away from the heated wall along the bubble axis. 


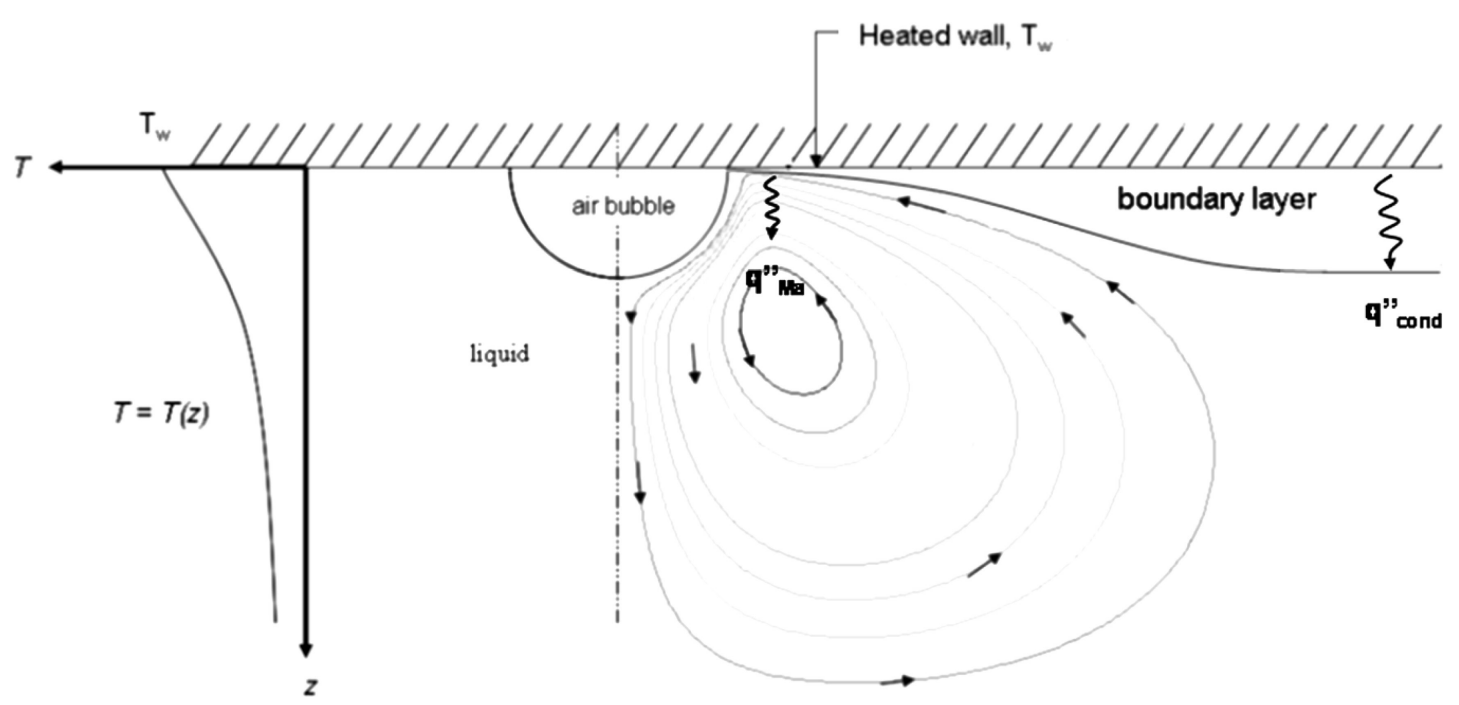

Figure 1 Surface tension induced flow around an isolated air bubble under a heated wall.

At some point, the fluid begins to change direction, recirculating the cooler bulk fluid toward the heated wall. At sufficiently low Marangoni numbers ( $\mathrm{Ma}<12,000)$ [4], this continued motion ts in a steady flow pattern of an axial jet-like fluid motion with major vortices on either side. Marangoni convection can thus influence the wall heat transfer by acting as a pump that transports hot fluid near the wall into the cooler bulk fluid.

A mechanistic model of boiling is necessary for accurate predictions of the associated heat transfer rates that are crucial for engineering many two-phase thermal systems and heat transfer devices. The range of technologies spans the microscale associated with the thermal management of micro-electronics to the much larger scale of steam power generation. Due to the comstill poorly understood, in the sense that predictive capabilities of theories and empirical correlations break down quickly once outside of the parameter range within which they were developed [5,6]. Of particular relevance to this work, the contribution of Marangoni convection to the heat transfer during boiling has historically been difficult to quantify since most experimental measurements are performed at terrestrial gravity where buoyant natural convection and Marangoni convection are impossible to decouple. Intriguingly, recent research has suggested that the 75 often overlooked Marangoni convection may play an important role in the heat transfer during phase change processes $[1,7,8]$ for both Earth and space applications. This was first suggested by McGrew et al. [9], who argued the theory that the high boiling heat transfer rates are due to intense vapor bubble disturbance of the liquid boundary layer close to the heating surface and bulk liquid disturbance due to bubble detachment from the surface. Experiments were performed in which the liquid was heated from above and cooled from below, with slowly increasing heat flux levels. McGrew et al. used tracer particles to examine the 85 flow pattern around air bubbles placed on the heating surface and vapor bubbles produced during boiling, and found that the flow patterns were identical for both air and vapor bubbles. McGrew et al. [9] concluded that Marangoni convection would occur around any bubble present in a region subjected to a temperature gradient. This convection, it was suggested, may also be a primary factor in the overall heat transfer mechanism in situations where bubbles remained affixed to a surface for relatively long periods of time.

With regard to the development of a mechanistic model of boiling that includes the influence of Marangoni convection, a further complication arises since the actual mechanism for the onset of thermocapillary flow during boiling remains somewhat elusive. This uncertainty arises largely because Marangoni convection has been observed experimentally in several microgravity boiling experiments $[10,11]$ and contradicts the notion that evaporation and/or condensation will create a uniform interface temperature and thus act to suppress the thermocapillary flow. However, recent evidence seems to indicate that during boiling Marangoni flow is caused by variation in the evaporation and condensation heat transfer coefficient at the vapor-liquid interface [12-14], which can possibly arise due to the presence of noncondensable gas $[10,12,15]$.

Lee and Merte [16] performed varying gravity boiling experiments using a gold film on a quartz substrate immersed in $\mathrm{R}-113$. Boiling behavior was analyzed under a wide range of heat fluxes and subcooling levels. Under saturated conditions at large heat fluxes, dryout of the heater surface was observed, but an increase in subcooling to $22^{\circ} \mathrm{C}$ resulted in steady nucleate boiling. A large bubble was seen to form above the surface, and remained constant in size due to a balance between condensation at the tip and evaporation at the base. Marangoni convection was seen to be an important additional mechanism, causing large vapor bubbles to be driven toward the heater surface. Heat transfer enhancements of up to $32 \%$ were obtained in microgravity when compared to terrestrial conditions.

The quest for further understanding of boiling behavior is explained by Kim and Benton [17], who highlight that boiling experiments to date have shown that stable subcooled boiling on 
flat plates is achievable in various gravity environments. Typically, some of the earlier results displayed a difference in heat transfer coefficients. As much of the earlier work was of a more qualitative than quantitative nature, further research was necessary to further develop the understanding of the boiling phenomenon. In subsequent investigations, where the heat transfer

130 coefficient was measured, the heater surfaces were often larger than or comparable to the bubble sizes, meaning that only an averaged measurement could be attained. Kim and Benton [17] stress the need for local heat transfer rates under and in the immediate vicinity of the bubbles on a heater surface as they grow and

135 depart from the heater surface. This data could then be correlated to visual observations of the dynamic bubble behavior, allowing the researcher to determine exactly when and where large amounts of heat were being transferred from the heater surface.

In order to better elucidate the contribution of Marangoni

140 convection to the flow and heat transfer during boiling, the simplified situation of a stationary gas bubble on a heated surface immersed in a liquid is typically investigated. Raake et al. [18] experimentally investigated temperature and velocity fields arising from surface tension flow caused by air bubbles. They

145 found that the convective transport mechanism operated along the entire bubble boundary for low convective flows-i.e., for flows with low Ma and relatively high Pr. Conversely, for highly convective flows where Ma is high compared with $\mathrm{Pr}$, it was established that the convective transport mechanism operated along a small part of the bubble surface near the wall. For their bubble diameter of $8 \mathrm{~mm}$, Raake et al. [18] found that an Ma $\approx 12,500$ coincided with the onset of an oscillatory mode that was axisymmetric. Increasing the temperature gradient caused the frequency of oscillation to increase.

Similar analysis was performed by Arlabosse et al. [7], who followed up their numerical work with an experimental investigation of Marangoni flow around an isolated bubble under a heated wall immersed in a liquid silicone oil layer. Contrary to the experimental observation of Raake et al. [18] mentioned pre-

160 viously, thermocapillarity was seen to be active along the entire contour of the bubble and, in the immediate vicinity of the bubble surface, was dominant over the buoyancy-driven convection. For higher temperature gradients, weak secondary counterrotating vortices resulting from the interaction of surface tension and

165 gravity forces were observed beneath the primary vortices. Arlabosse et al. [7] concluded that the effects were coupled under terrestrial conditions: The thermocapillarity destroys the stable temperature stratification, which causes natural convection as a secondary effect. The secondary vortices then reduce the size

170 of the main cells. From the results obtained, it was determined that the ratio of heat transfer by Marangoni convection to that solely by conduction was well correlated by the relation

$$
\frac{q_{\text {Ma }}^{\prime \prime}}{q_{\text {cond }}^{\prime \prime}}=1+0.00841 M a^{0.5}
$$

Recently, the empirically based heat transfer model presented by Petrovic et al. [19] has successfully quantified the contributions of buoyant natural convection and Marangoni convection for the situation in which gas bubbles were situated on an upward facing heated surface immersed in water. Here, Marangoni convection was determined to be a dominant heat transfer mechanism. For air bubbles of similar size the measurements were in excellent agreement with the relationship proposed by Arlabosse et al. [7].

Apart from the few studies mentioned earlier, most of what is understood about thermocapillary flows around bubbles is concerned with the fluid mechanics. For example, much of the experimental and numerical work focuses on flow analysis techniques, flow imaging of thermocapillary convection, and numerical predictions of the flow field [6,7,20-23], and far less is understood about the coupling of the fluid mechanics and the heat transfer. In this study an attempt is made to quantify the contribution of Marangoni convection to the heat transfer for the simplified case of a bubble affixed to a downward facing heated surface immersed in silicon oil. For a fixed Ma $=915$ and $\operatorname{Pr}=83$, the influence of gravity on the fluid dynamics and heat transfer is elucidated for the range of gravity level between $0 g$ and $1 g$.

\section{NUMERICAL METHOD}

For thermocapillary convection around a bubble of radius $\mathrm{R}_{\mathrm{b}}$ within a channel of height $\mathrm{H}$, subject to gravitational forces, the mass and heat transport processes are characterized by the Prandtl, Marangoni, and Rayleigh numbers. The Prandtl number represents the ratio of viscous to thermal diffusivity in the fluid. Since variations in fluid viscosity and diffusivity are neglected in this study, the Prandtl number can be assumed constant. The Marangoni number represents the ratio of surface tension forces to viscous forces, and is often seen in different forms depending on the particular problem. In this study, the bubble radius was selected as the characteristic length, and the definition of the Marangoni number was chosen to include the depth of the liquid layer. This form of the dimensionless number is consistent with that of $[6,7,20,24,25]$. The Marangoni number for this study is fixed at 915 . The Rayleigh number represents the ratio of buoyant to viscous forces in the fluid. When the Rayleigh number is below the critical value for a particular fluid, viscous forces are dominant and heat transfer is therefore primarily by conduction; when it exceeds the critical value, buoyancy forces are strong enough to overcome viscosity and heat transfer is primarily by convection. The Rayleigh number for this study is modified by changing the magnitude of gravitational acceleration between simulations. These dimensionless numbers are defined as follows:

$$
\operatorname{Pr}=\frac{v}{\alpha}
$$

$$
\begin{aligned}
M a & =-\left(\frac{\partial \sigma}{\partial T}\right) \times \frac{\left(T_{h}-T_{c}\right)}{\mu \alpha} \times \frac{R_{b}^{2}}{H} \\
R a & =\frac{g \beta\left(T_{h}-T_{c}\right) R_{b}^{4}}{v \alpha H}
\end{aligned}
$$


The commercial code FLUENT release 6.2.16 was utilized to solve the governing equations. From calculations of the Reynolds number, it was known that the resulting flow would be laminar. The segregated solver was selected on the basis of computing power. The numerical scheme adopted was secondorder upwind. Grid independence was obtained by increasing the number of structurally arranged quadrilateral cells from 1260 to 81,000 . To validate the numerical approach undertaken in this study, some results were compared with experimental data pro230 vided by Arlabosse et al. [7]. The validation simulations are described in further detail in [26]. The agreement between the simulations and the experimentally measured nondimensional interface velocities is quite good. Arlabosse et al. [7] also measured the increase in the average heat flux over a heat flux sensor situated on the wall opposite to that of the bubble. Comparison between their experimental data and the numerically simulated data was found to be satisfactory.

The governing equations that were solved were the steadystate energy, continuity, and momentum equations.

Energy:

$$
v_{z} \frac{\partial T}{\partial z}+v_{r} \frac{\partial T}{\partial r}=\alpha\left(\frac{\partial^{2} T}{\partial z^{2}}+\frac{1}{r} \frac{\partial T}{\partial r}+\frac{\partial^{2} T}{\partial r^{2}}\right)
$$

Continuity:

$$
\frac{\partial}{\partial z}\left(\rho v_{z}\right)+\frac{\partial}{\partial r}\left(\rho v_{r}\right)+\frac{\rho v_{r}}{r}=0
$$

Axial momentum:

$$
\begin{aligned}
\frac{1}{r} \frac{\partial}{\partial z} & \left(r \rho v_{z}^{2}\right)+\frac{1}{r} \frac{\partial}{\partial r}\left(r \rho v_{z} v_{r}\right) \\
& =-\frac{\partial p}{\partial z}+\frac{1}{r} \frac{\partial}{\partial z}\left[r \mu\left(2 \frac{\partial v_{z}}{\partial z}-\frac{2}{3}(\nabla \cdot \vec{v})\right)\right] \\
& +\frac{1}{r} \frac{\partial}{\partial r}\left[r \mu\left(\frac{\partial v_{z}}{\partial r}+\frac{\partial v_{r}}{\partial z}\right)\right]+F_{B}
\end{aligned}
$$

where

$$
\begin{aligned}
F_{B} & =-\rho_{0} \beta g\left(T-T_{0}\right) \\
\nabla \cdot \vec{v} & =\frac{\partial v_{z}}{\partial z}+\frac{\partial v_{r}}{\partial r}+\frac{v_{r}}{r}
\end{aligned}
$$

Radial momentum:

$$
\begin{aligned}
\frac{1}{r} \frac{\partial}{\partial z} \quad & \left(r \rho v_{z} v_{r}\right)+\frac{1}{r} \frac{\partial}{\partial r}\left(r \rho v_{r}^{2}\right) \\
= & -\frac{\partial p}{\partial r}+\frac{1}{r} \frac{\partial}{\partial z}\left[r \mu\left(\frac{\partial v_{r}}{\partial z}+\frac{\partial v_{z}}{\partial r}\right)\right] \\
+ & \frac{1}{r} \frac{\partial}{\partial r}\left[r \mu\left(2 \frac{\partial v_{r}}{\partial r}-\frac{2}{3}(\nabla \cdot \vec{v})\right)\right] \\
& -2 \mu \frac{v_{r}}{r^{2}}+\frac{2}{3} \frac{\mu}{r}(\nabla \cdot \vec{v})
\end{aligned}
$$

In accordance with previous numerical investigations on 245 Marangoni convection, the following assumptions were made in the analysis:

- Motion is two-dimensional (2D) axisymmetric in cylindrical coordinates.

- Bubble can be represented by a rigid hemispherical interface. 250

- Heat flux is zero at the bubble interface.

- Boussinesq approximation accounts for any changes in density due to temperature gradients.

- Other physical properties are constant $(\mu, k \neq f(T, t \ldots)$ etc. $)$.

From experimental observations $[2,6,7]$ it is known that the flow field is symmetric about the bubble vertical axis. Furthermore, results by Kassemi and Rashidnia [4] show the onset of unsteady oscillatory and three-dimensional (3D) flow occurring at a critical Marangoni number of approximately 12,000. Since the Marangoni number in this study is considerably lower and fixed at 915, the steady-state, 2D axisymmetric form of the governing equations is sufficient to model the problem accurately. The action of gravitational forces gives rise to hydrostatic pressure, which would act to deform the bubble shape. However, results obtained by Arlabosse et al. [7] indicate that for the same fluid, albeit at a higher Prandtl number, up to a radius of $1.3 \mathrm{~mm}$, the bubble maintains a spherical shape in a geometrical configuration similar to this study. The bubble radius for this investigation is $1 \mathrm{~mm}$. Furthermore, in [7] the contact angle increased from $52^{\circ}$ to $71.5^{\circ}$ for bubbles of equivalent radius $2.0 \mathrm{~mm}$ and $1.5 \mathrm{~mm}$, respectively. Extrapolating to $1.0 \mathrm{~mm}$ would result in a contact angle of $90^{\circ}$. This suggests that the hemispherical shape may even be valid in a terrestrial gravity field.

The adiabatic assumption along the bubble interface is also consistent with the Marangoni heat transfer regime discovered by Petrovic et al. [19] where air bubbles caused a significant enhancement in the heat transfer even when the heated wall temperature was below the saturation temperature. The work is also qualitatively comparable to the situation of gas-saturated liquids, for example, the experimental results of Henry et al. [10].

For many natural convection flows it is possible to obtain faster convergence using the Boussinesq model rather than solving the problem with fluid density as a function of temperature. This model treats density as a constant value in all solved equations, except for the buoyancy term in the momentum equation.

$$
\left(\rho-\rho_{0}\right) g \approx \rho_{0} \beta\left(T-T_{0}\right) g
$$

In the preceding equation, $\rho_{0}$ is the (constant) reference density of the flow, $T_{0}$ is the operating or reference temperature, and $\beta$ is the thermal expansion coefficient. Equation (11) is obtained by using the Boussinesq approximation (Eq. [12]) to eliminate $\rho$ from the buoyancy term. This approximation is accurate as long as changes in actual density are small; specifically, the Boussinesq approximation is valid when $\beta *\left(\mathrm{~T}-\mathrm{T}_{0}\right)<<1$.

$$
\rho=\rho_{0}(1-\beta \Delta T)
$$




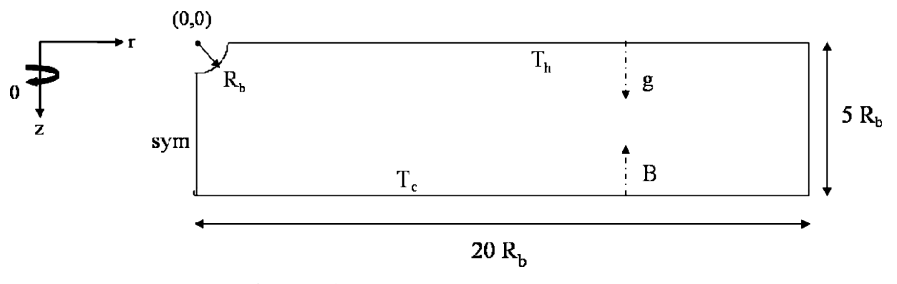

Figure 2 Model domain schematic.

The geometry was chosen to approximate that seen in Arlabosse et al. [7]. The model domain is shown in Figure 2. A bubble of radius $1.0 \mathrm{~mm}$ is placed at the center of the coordinate plane. The vertical wall denoted "sym" signifies the axis of symmetry. Two horizontal walls are separated by a distance $\mathrm{H}$, which is equivalent to five times the bubble radius. The upper wall is no slip, constant temperature:

$$
\vec{v}=0, \quad T=T_{0}
$$

and is maintained at the temperature of $350 \mathrm{~K}$ for all simulations. The lower horizontal wall is also no slip and constant temperature:

$$
\vec{v}=0, \quad T=T_{0}
$$

and the temperature of this wall is maintained at the temperature of $300 \mathrm{~K}$ for all simulations. The vertical wall placed 20 bubble radii from the bubble has a no-slip, adiabatic condition.

$$
\vec{v}=0, \quad \vec{n} \cdot(k \nabla T)=0
$$

The bubble surface is comprised of a slip condition, and an adiabatic condition. The bubble is represented by a boundary upon which a Marangoni stress is directly applied. This equation (Eq. [17]) relates the shear stress on the surface to the temperature derivative of surface tension.

$$
\begin{aligned}
\vec{n} \cdot \vec{v} & =0, \quad \vec{n} \cdot(k \nabla T)=0 \\
\vec{\tau} & =\frac{d \sigma}{d T} \nabla T
\end{aligned}
$$

Solutions were carried out for gravitational acceleration levels of $0 \mathrm{~g}, 0.01 g, 0.1 \mathrm{~g}, 0.25 \mathrm{~g}, 0.5 \mathrm{~g}, 0.75 \mathrm{~g}$, and $1 \mathrm{~g}$, where $g$ represents the traditional earth gravitational acceleration of $9.81 \mathrm{~m}$ $\mathrm{s}^{-1}$. This corresponded to Rayleigh numbers of approximately $0,1.6,15.6,38.9,77.8,116.7$, and 155.6 , respectively. A temperature difference of $50 \mathrm{~K}$ was imposed on the system, which corresponded to a Marangoni number of 915. The test fluid was selected to have the same properties as silicone oil of kinematic viscosity $7.5 \mathrm{cSt}$. The relevant physical properties are given in Table 1.
Table 1 Properties of silicone oil, 7.5cSt

\begin{tabular}{lccccc}
\hline $\begin{array}{l}\rho \\
\left(\mathrm{kg} / \mathrm{m}^{3}\right)\end{array}$ & $\begin{array}{c}\mathrm{k} \\
\left(\mathrm{m}^{2} / \mathrm{s}\right)\end{array}$ & $\begin{array}{c}\mathrm{k} \\
(\mathrm{W} / \mathrm{m}-\mathrm{K})\end{array}$ & $\begin{array}{c}\beta \\
(1 / \mathrm{K})\end{array}$ & $\begin{array}{c}\mathrm{C}_{\mathrm{p}} \\
(\mathrm{J} / \mathrm{kg}-\mathrm{K})\end{array}$ & $\begin{array}{c}\mathrm{d} \sigma / \mathrm{dT} \\
(\mathrm{N} / \mathrm{m}-\mathrm{K})\end{array}$ \\
\hline 930 & $7.5 \mathrm{e}-6$ & 0.125 & $1.08 \mathrm{e}-3$ & 1480 & $-5.8 \mathrm{e}-5$ \\
\hline
\end{tabular}

\section{RESULTS AND DISCUSSION}

\section{Effect of Gravity Level on Flow and Temperature}

Figure 3, a to g, shows the temperature and velocity fields for different gravity levels. The flow in each image consists of a major (anticlockwise) vortex, which recirculates the colder fluid from the lower regions, pulling it toward the hot wall toward the triple contact point, stripping heat from the wall in the process. This redistribution of colder fluid causes the temperature contours to compress toward the heated surface near the bubble. The fluid is accelerated as it is drawn toward the bubble, before being pulled along the interface itself. The fluid continues to moves toward the bubble centerline and ultimately leaves the bubble as a jet that decelerates with increasing distance from the bubble. The liquid jet, in flowing away from the bubble, causes the temperature contours to project outward, transporting hot fluid into the cooler bulk, and toward the cold surface. This type of flow pattern has been seen in various experiments [6,7,20,25]. By applying the same streamline range and grayscale to each plot, it is evident that with increased gravitational acceleration, and therefore increased buoyancy, the thermocapillary effect becomes increasingly confined to a region closer to the bubble. In particular, the jet-like flow stemming from the bubble apex becomes restricted by the buoyant forces opposing it. The mean diameter of the major vortex decreases with increasing gravity level, and secondary vortices develop that highlight the presence of buoyant forces. These secondary vortices have also been observed by Arlabosse et al. [24].

Figure 4a shows the interfacial velocity profile around the bubble for different gravity levels. For clarity of explanation, some of the data has been omitted. Angles are computed from the heated wall to the centreline of the bubble. From the figure it is evident that the gravity level has a notable influence on the velocity profile. The largest velocities are found for the $0 \mathrm{~g}$ case. Up to an angle of approximately $7^{\circ}$, i.e., near the triple contact line, all curves lie on the same curve, with slight deviation from this point until the maximum velocity is reached. The location corresponding to maximum velocity for all test cases was found to be almost independent of gravity level. However, from this point on, the gravity level has a significant impact on the velocity profiles. At lower gravity levels $(<0.5 \mathrm{~g})$ in the range $20^{\circ} \leq \theta \leq$ $90^{\circ}$ the interfacial velocity profiles were found to have similar slopes. At the higher gravity levels $(>0.5 g)$, the behavior changed in the range $40^{\circ} \leq \theta \leq 90^{\circ}$. Since the driving force for Marangoni convection must be the interfacial temperature gradient, the temperature profiles along the bubble were analyzed in an effort to understand the phenomenon. As shown in Figure 

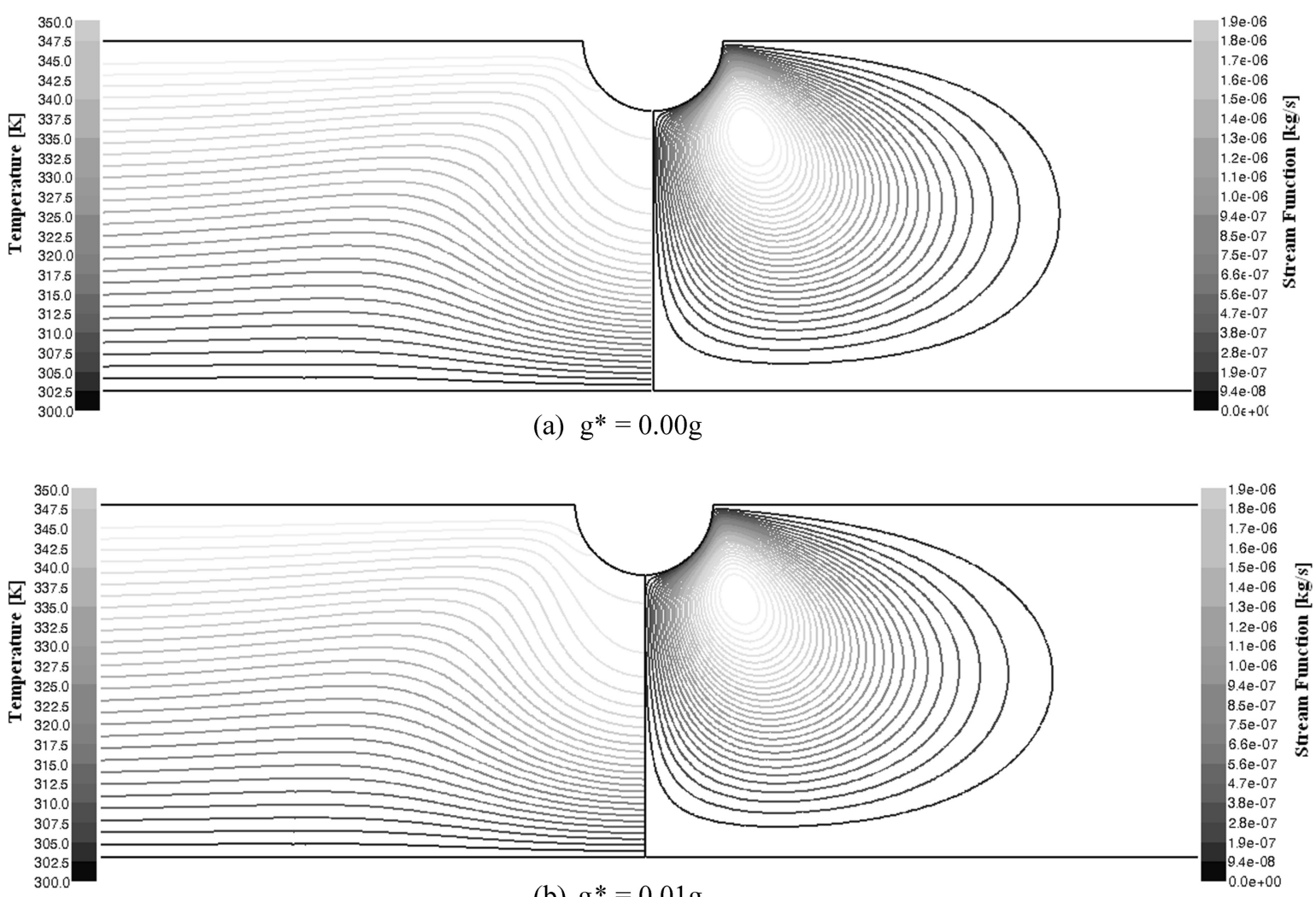

(b) $\mathrm{g} *=0.01 \mathrm{~g}$
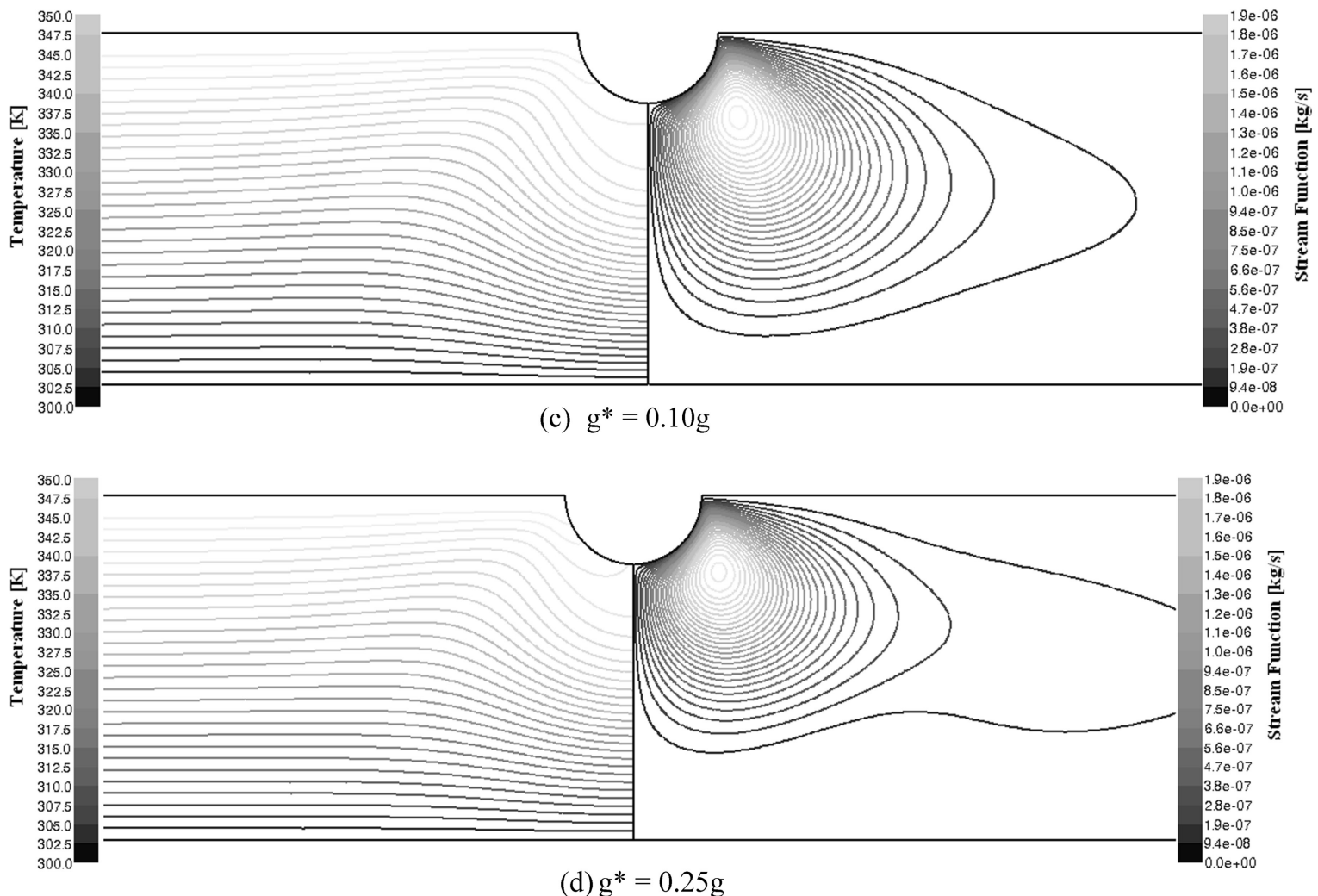

Figure 3 Contours of temperature (left) and stream function (right) for varying gravity levels. (Continued) 


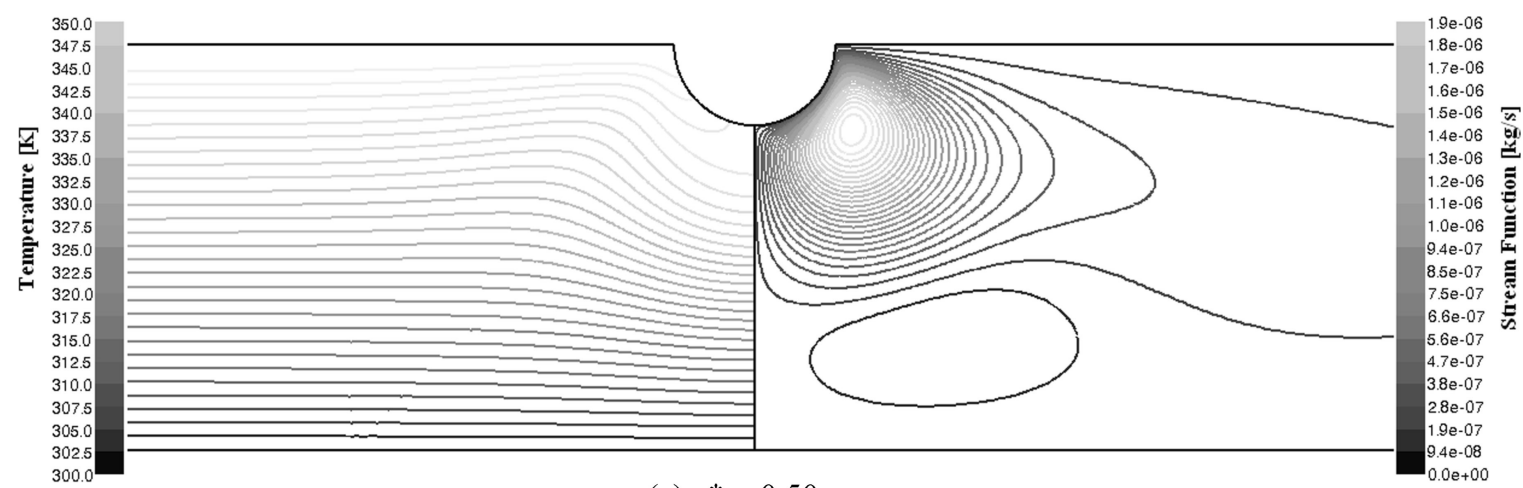

(e) $g^{*}=0.50 \mathrm{~g}$

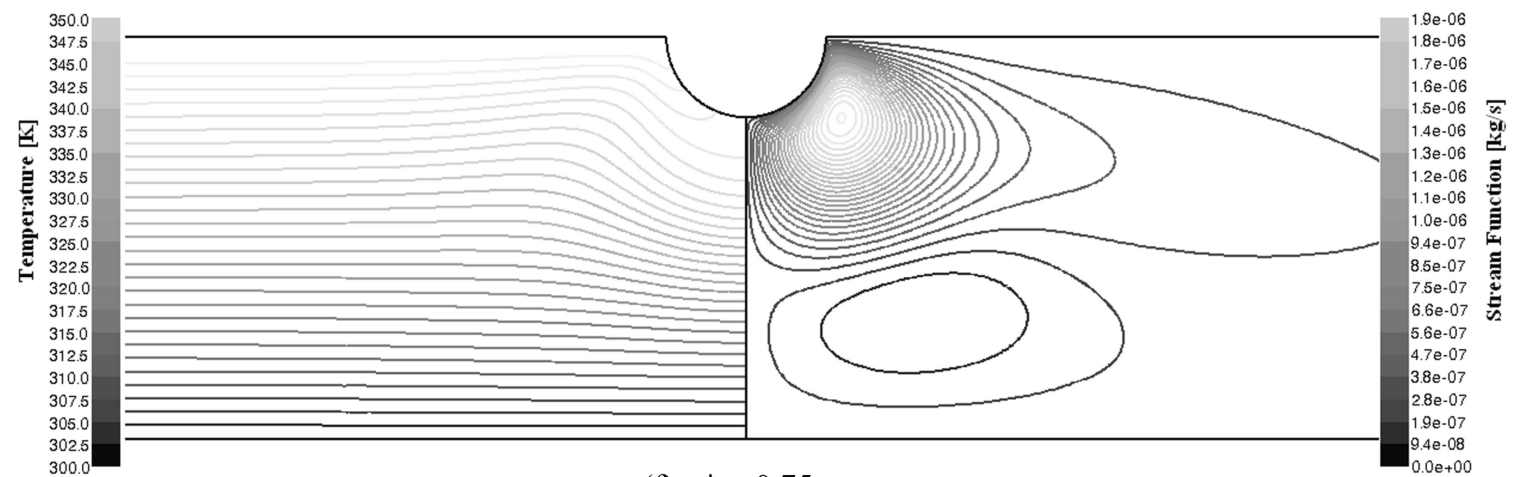

(f) $g^{*}=0.75 g$

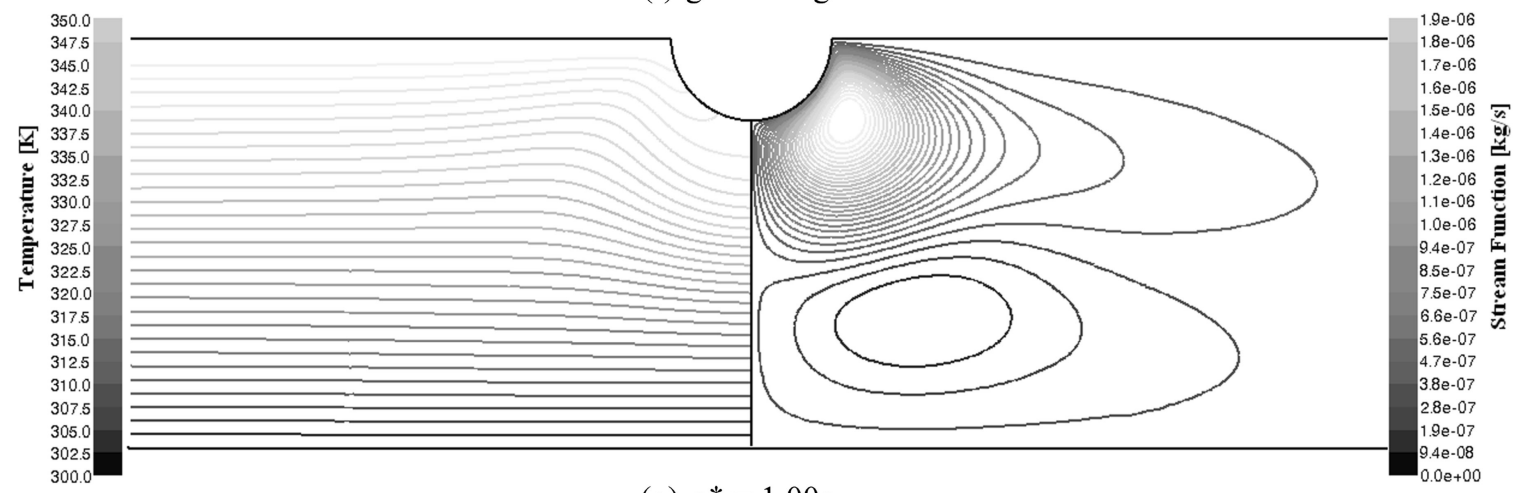

(g) $\mathrm{g}^{*}=1.00 \mathrm{~g}$

Figure 3 (Continued)

$4 \mathrm{~b}$, the temperature profiles display characteristics similar to the velocity profiles. Larger gravity levels deviate in the range $40^{\circ} \leq \theta \leq 90^{\circ}$. Greater gravity levels display a greater overall interfacial temperature gradient, meaning that the increased buoyancy pushes colder fluid nearer the bubble and generates a larger driving force over a small portion of the interface.

\section{Effect on Heat Transfer}

In an effort to quantify the total amount of heat transfer within their system, Petrovic et al. [19] tried to analyze what was happening on the heater surface during their nucleate pool boiling experiments. At any time, there may be any two bubbles in close proximity on the heater surface. Typically the bubbles will be of different radius. Each bubble will have its own radius of enhanced heat transfer caused primarily by Marangoni convection. This may depend on the bubble size and may therefore be different for each bubble. Depending on the distance between bubbles, there may also be natural convection. Petrovic et al. [19] wanted to calculate the distance over which the bubble affected the wall heat transfer, and estimated a value of two bubble radii at the time. While realizing the need for further study on the topic, Petrovic et al. [19] attempted to perform an energy balance on the system using an approach proposed by Judd and Hwang [27]. This approach, shown in Eq. (18), divides the total rate of heat transfer into its constitutive parts-namely, natural convection and Marangoni convection. The primary unknown in this expression was the area affected by the bubble, $A_{b, i}$. This study attempts to quantify the affected area for heat transfer for 


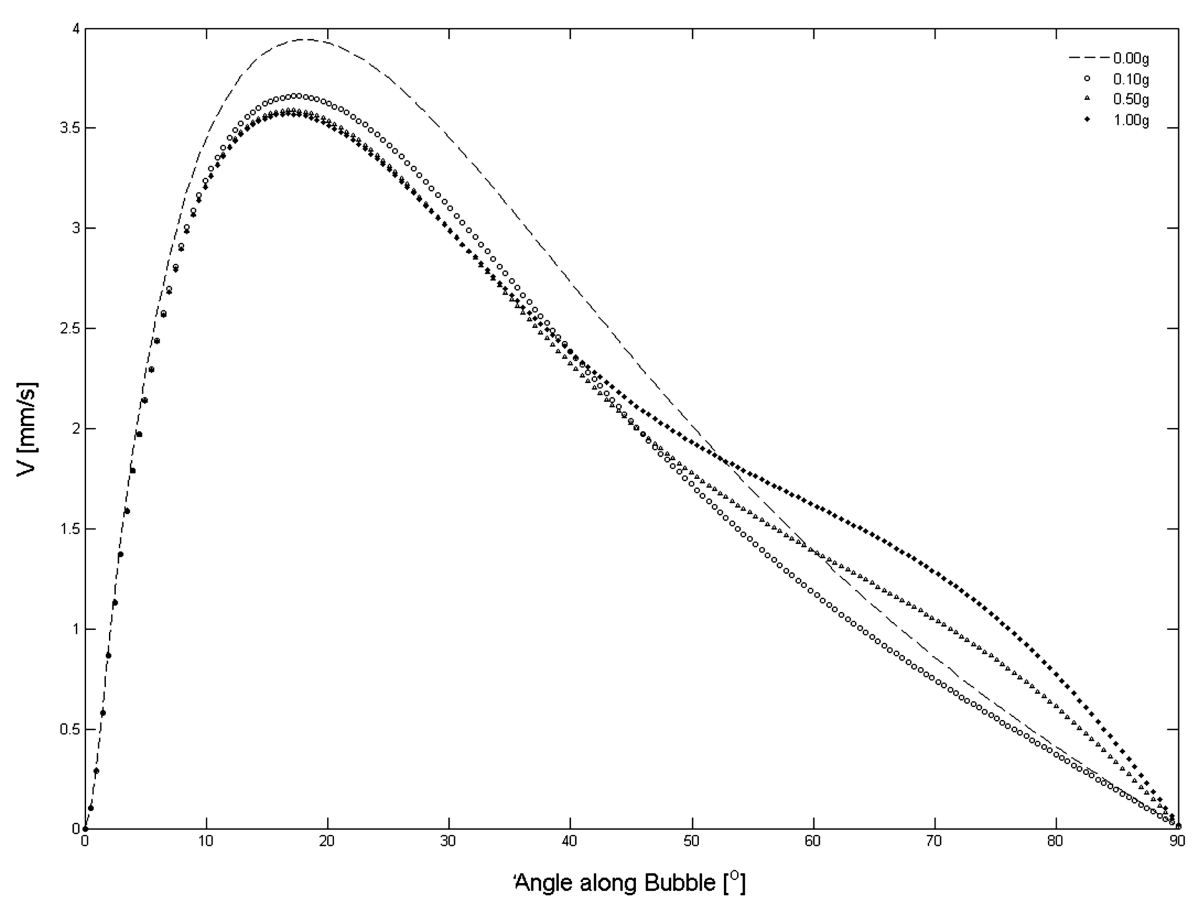

(a) Interfacial fluid velocities

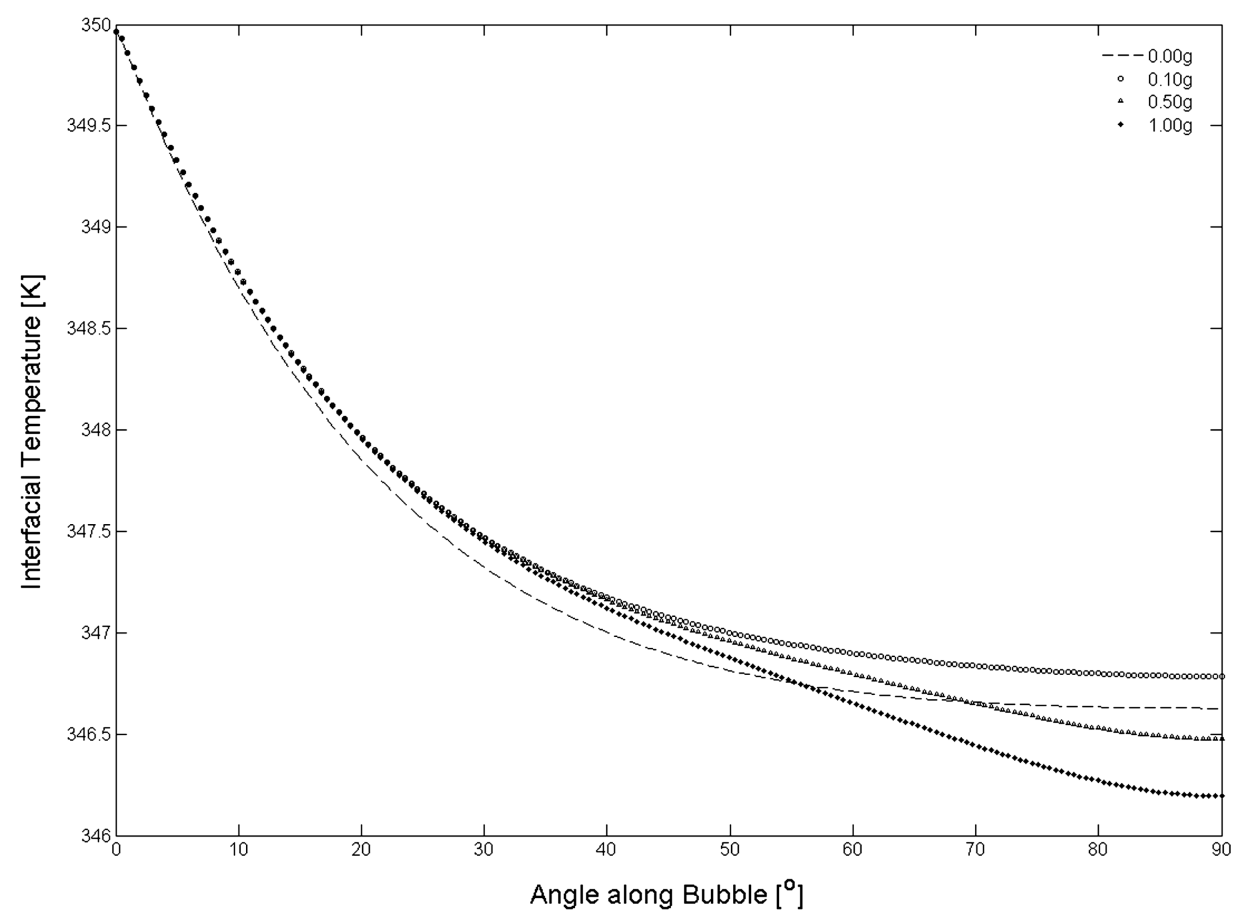

(b) Interfacial fluid temperatures

Figure 4 Interfacial velocity (a) and temperature (b) profiles for different gravity levels.

an isolated bubble on a heated surface, which can be equated to the $i$ th bubble in Eq. (18).

$$
q_{\mathrm{tot}}^{\prime \prime} A_{\mathrm{tot}}=q_{\mathrm{nc}}^{\prime \prime} A_{\mathrm{nc}}+\sum_{i=1}^{N_{b}} q_{\mathrm{Ma}, \mathrm{i}}^{\prime \prime} A_{\mathrm{b}, \mathrm{i}}
$$

Figure 5 depicts the concept of a defined area of heat transfer. For clarity of explanation, the image is displayed with the bubble on the heated wall at the bottom as opposed to the configuration under investigation in this study. A typical hot wall heat flux profile is provided on the top right-hand side. The 


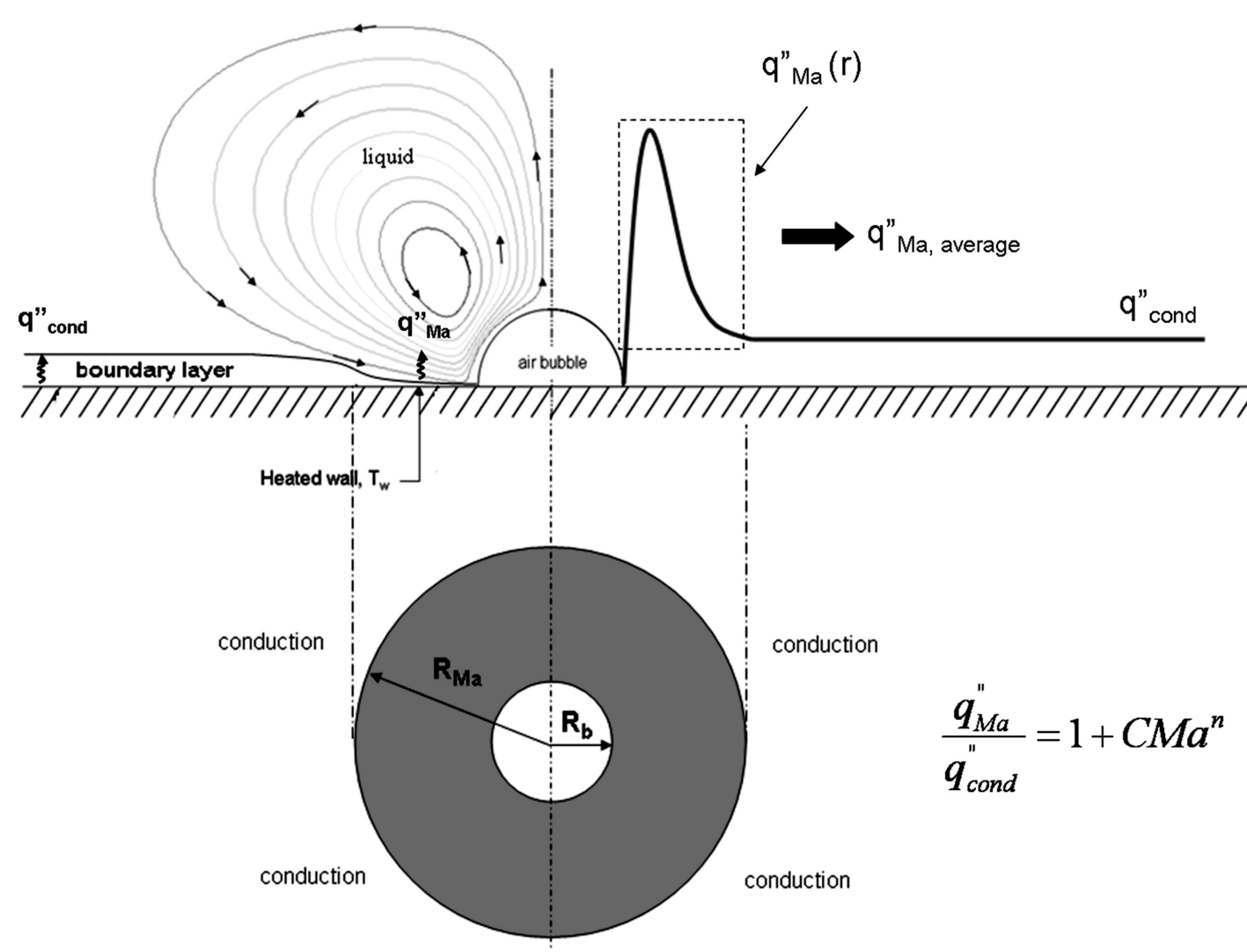

Figure 5 Marangoni area of enhancement for the bubble affixed to a heated wall.

Marangoni effect occurs over some range measured outward from the center of the bubble. In the immediate vicinity of the

bubble, the heat transfer increases dramatically, greatly exceeding that by conduction alone. At some point thermocapillarity ceases to have effect and conduction and/or natural convection are the sole modes of heat transfer.

The reduction of local heat transfer caused by increased gravity levels is best visualized by plotting the heat transfer enhancement due to Marangoni convection. For this study, heat transfer due to thermocapillary convection is defined differently from that of Petrovic et al. [19] (Eq. [18]). This factor is expressed as the ratio of heat transfer in the presence of Marangoni convection to that by pure molecular diffusion, where

$$
q_{\mathrm{Ma}}^{\prime \prime}=q_{\mathrm{tot}}^{\prime \prime}=q_{\mathrm{st}}^{\prime \prime}+q_{\mathrm{nc}}^{\prime \prime}+q_{\mathrm{cond}}^{\prime \prime}
$$

Figure 6 illustrates the normalized heat flux profiles along the hot and cold walls caused by Marangoni convection for each of the gravity levels simulated. Simulations were performed over a similar domain under zero-gravity conditions in the absence of the bubble to predict the heat transfer due to pure molecular diffusion and establish a "baseline" to which subsequent conditions can be compared. It was found that the heat flux along the channel walls was constant in the absence of the bubble, which is consistent with analytical predictions. Figure 6a shows the hot wall enhancement factor for increasing gravity levels. The maximum heat flux values for all profiles occur in the interval $1.3<\mathrm{r} / \mathrm{R}_{\mathrm{b}}<2$. As expected, the maximum enhancement factor decreases with increasing gravity level, but only slightly, with a maxima range of $60-67 \%$. Interestingly, it is the range of enhancement that shows the greatest response to buoyant forces. It is clear that with larger values of gravitational acceleration, the area of enhancement decreases. For the cold wall shown in Figure 6b, the range of enhancement remains approximately constant at four bubble radii. However, the enhancement factor changes dramatically between test cases. This is consistent with Figure 3, a to g, previously shown. The jet-like flow is inhibited by buoyancy forces acting upward, pushing the warm fluid to a region closer to the bubble. In effect, the vortex is squeezed from below. Thus, with increasing gravity level the warmer fluid does not reach the cold wall to provide the same level of heat transfer. For the larger gravity levels, almost no enhancement is seen at the cold wall.

Strangely, under zero gravity conditions the enhancement factor can be seen to briefly fall below the value for pure conduction. This phenomenon may possibly be explained by analyzing the temperature contours over the whole domain, shown in Figure 7. The region directly beneath the jet will have the best heat transfer, as the fluid velocity is relatively high. This high heat transfer area can be correlated to the small band of low temperature, a kind of thermal boundary layer, denoted $\delta_{1}$. Heat transfer occurs at the lower wall; the fluid loses heat and is recirculated by the main vortex. This results in a larger thermal boundary layer over an area corresponding to the dip in enhancement seen 


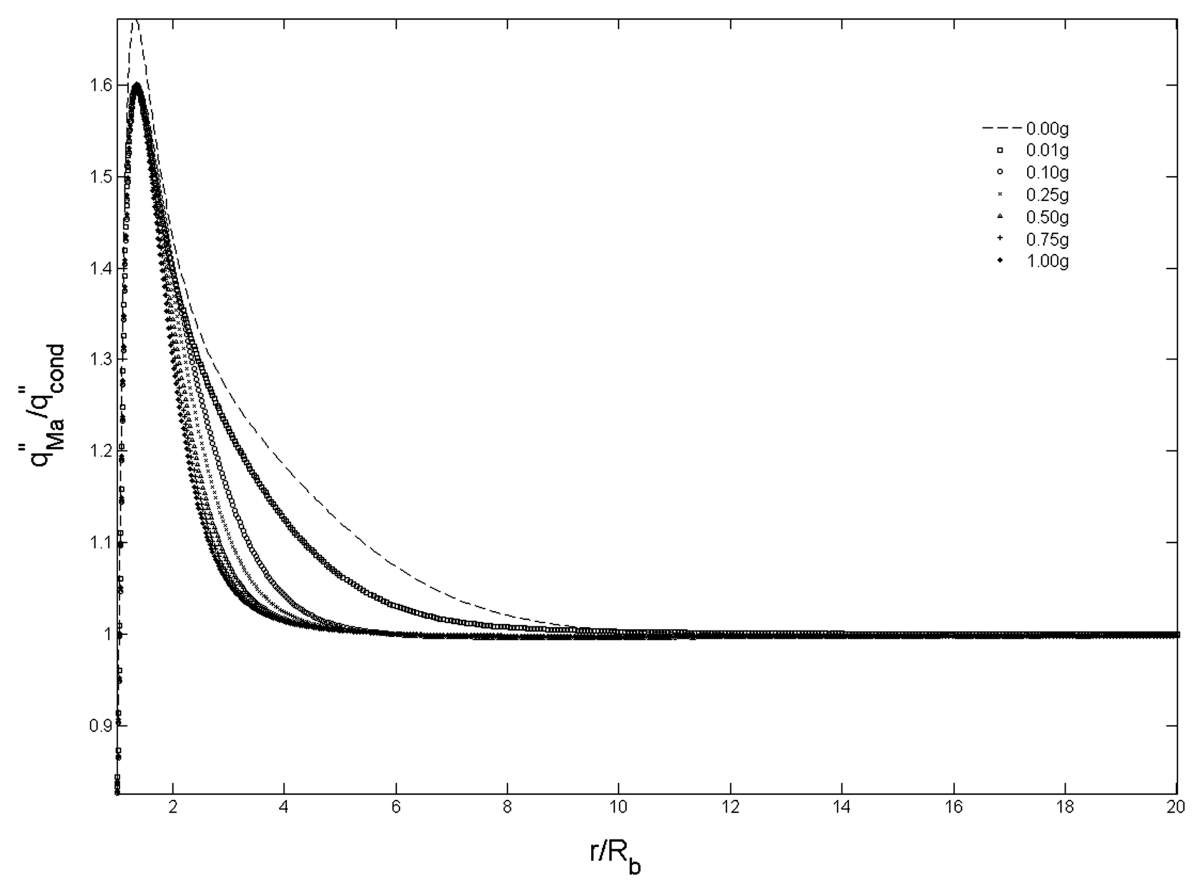

(a) Hot wall

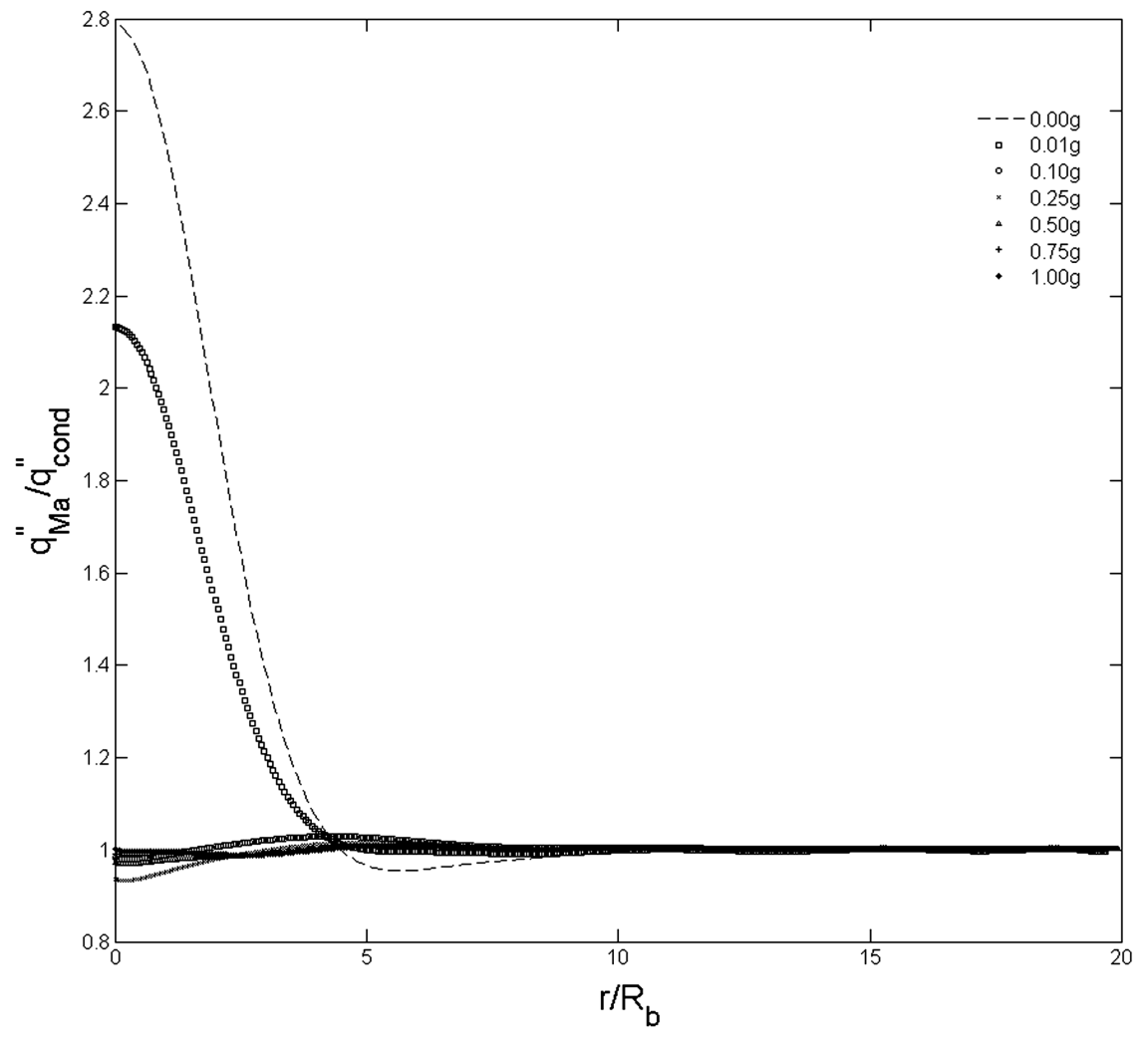

(b) Cold wall

Figure 6 Surface heat flux enhancement. 


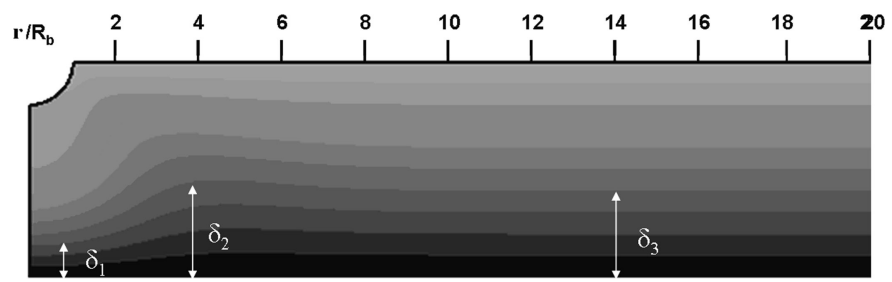

Figure 7 Temperature contours for the zero gravity simulation.

in Figure 6b. This "bump" is denoted $\delta_{2}$. Beyond this region, the vortex ceases to have effect on the lower wall heat transfer and the boundary layer thickness falls to its pure conduction value, denoted by $\delta_{3}$.

An enhancement range may be defined in terms of a radius measured outward from the center point of the bubble. The enhancement criterion is the location at which the heat transfer due to Marangoni convection falls to within 5\% of the value due to conduction only, or equivalently,

$$
R_{\text {enhancement }}=\left.\frac{r}{R_{b}}\right|_{1.05 \times q_{\text {cond }}^{\prime \prime}}
$$

Using Eq. (20), it is possible to determine the individual enhancement radii and corresponding area for each test case, at both the hot and cold walls. The results for enhancement radius are presented in Figure 8.

In a previous study under zero-gravity conditions with increasing Marangoni number [26], it was shown that the size of the primary vortex was independent of $\mathrm{Ma}$, but rather the
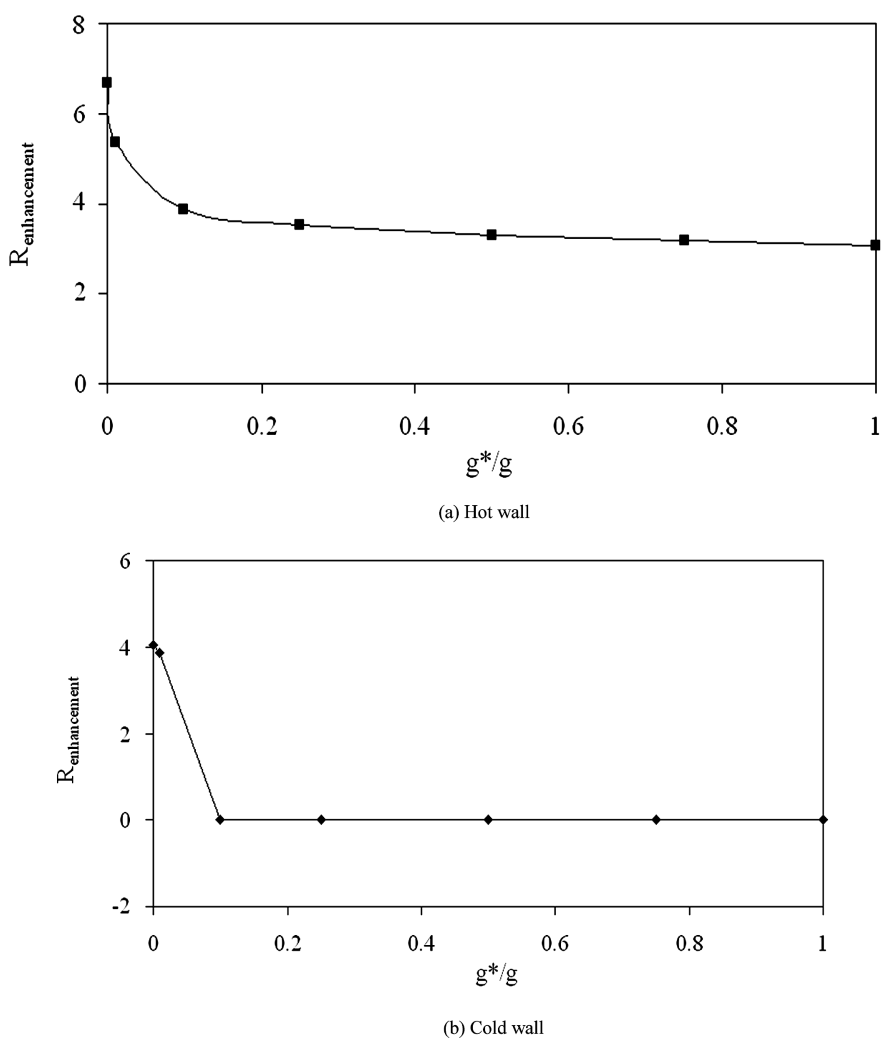

Figure 8 Enhancement radius vs. gravity level. intensity of the vortex increased with increasing Ma, which in turn increased wall heat transfer. For this study at fixed Ma, the influence of gravity level on the primary vortex is clear. Figure 8a shows that increasing gravity levels cause a decrease in the hot surface radius of enhancement. As buoyancy increases, the size of the primary vortex decreases, being squeezed to a region closer to the bubble. Therefore, the redistribution of the colder fluid takes place over a progressively smaller area of the domain, and its effect upon the hot surface diminishes. For the cold wall shown in Figure $8 \mathrm{~b}$, maximum heat transfer is achieved at the region exposed to the jet-like flow, with greater fluid velocities found here than for any other location on the cold wall. In accordance with previous images, the cold wall shows the greatest response to increasing gravity levels. Indeed, at gravity levels greater than $0.01 \mathrm{~g}$, there is no discernable cold wall enhancement radius as defined by Eq. (20). However, there remains a region where the local heat flux values exceed the pure conduction values, but not by a sufficient amount to meet the criteria for Eq. (20). Increased buoyancy pushes the primary vortex closer to the bubble, and the jet is likewise inhibited. Therefore, the warm fluid does not reach the cold wall, and this energy is distributed throughout the domain by the secondary vortical structures. Since each gravity level has its own radius and corresponding area of enhancement, it is useful to define any improvement in heat transfer over a constant range. For this investigation, the test case with zero gravitational acceleration forms the base case scenario. Therefore, an effective radius and
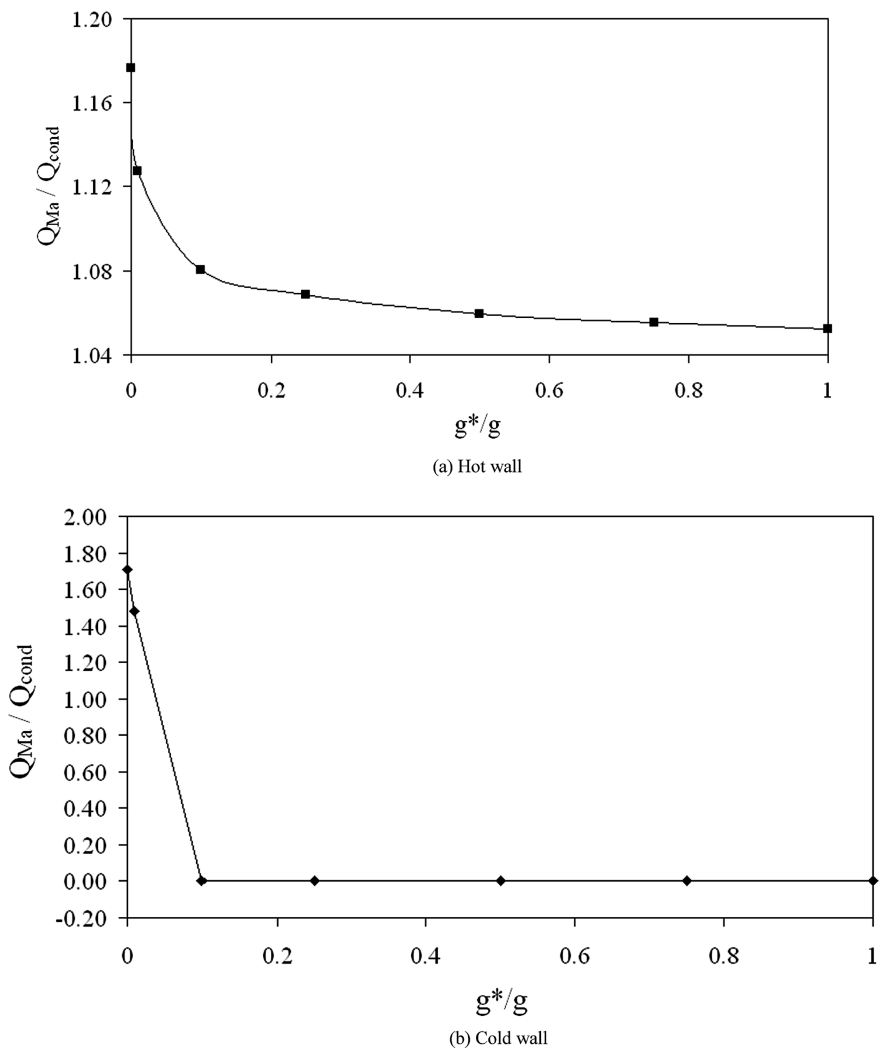

Figure 9 Total rate of heat transfer enhancement vs. gravity level.

vol. 30 no. 132009
470 
effective area of enhancement may be defined such that:

$$
\begin{aligned}
& R_{\mathrm{eff}, \mathrm{hw}}=7 R_{b} \quad A_{\mathrm{eff}, \mathrm{hw}}=\pi\left(R_{\mathrm{eff}, \mathrm{hw}}^{2}-R_{b}\right) \\
& R_{\mathrm{eff}, \mathrm{cw}}=4 R_{b} \quad A_{\mathrm{eff}, \mathrm{cw}}=\pi R_{\mathrm{eff}, \mathrm{cw}}^{2}
\end{aligned}
$$

With these data, the total rate of heat transfer enhancement through the effective area of enhancement is provided in Figure 9.

Figure 9a shows that for the zero gravity case an increase in hot wall heat transfer of $18 \%$ is achievable, which is significant. Under terrestrial conditions, an improvement of $5 \%$ is predicted. At Marangoni numbers greater than 915, further improvements may be attainable. The rate of heat transfer enhancement at the cold wall can be seen in Figure 9b. Note that at Earth gravity, there is no range of enhancement defined by Eq. (20). Figure $9 \mathrm{~b}$ describes the notable improvement in heat transfer at the cold wall by thermocapillary convection, with enhancement of up to $70 \%$ for the zero gravity case. However, cold wall heat transfer is affected dramatically by increasing gravity level, and for this configuration under terrestrial conditions, no cold wall heat transfer enhancement was calculated.

\section{CONCLUSIONS AND OUTLOOK}

The influence of the magnitude of gravitational acceleration on the velocity profile along the bubble interface and on the location of maximum velocity was analyzed. It was found that the gravity level affected the velocity profile by modifying the interfacial temperature gradient, but that the location of maximum velocity was almost independent of gravity level. The increase in heat flux on the wall to which the bubble is attached was computed. It was shown that heat flux due to thermocapillary convection was significantly better than heat flux due solely to conduction up to a distance of seven bubble radii for the zero gravity case, but increased gravity levels cause a reduction in the effective radius of enhancement. The increase in heat flux on the wall opposite to which the bubble is attached was also measured, with notable improvement in heat transfer up to four bubble radii for the zero gravity case.

Further simulations will be carried out at a greater range of Marangoni numbers. The domain height, which was fixed at 5 $\mathrm{mm}$ for this study, can be altered. By keeping the temperature difference across the domain constant and varying fluid viscosity, it is possible to explore the effect of the Prandtl number.

535 The influence of bubble shape is also an important aspect that requires a detailed investigation.

\section{NOMENCLATURE}

$$
\mathrm{A}_{\mathrm{b}} \quad \text { area affected by bubble }\left[\mathrm{m}^{2}\right]
$$

\begin{tabular}{|c|c|}
\hline $\mathrm{A}_{\mathrm{eff}, \mathrm{hw}}$ & effective area, hot wall $\left[\mathrm{m}^{2}\right]$ \\
\hline $\mathrm{A}_{\mathrm{nc}}$ & area affected by natural convection $\left[\mathrm{m}^{2}\right]$ \\
\hline $\mathrm{A}_{\text {tot }}$ & total area $\left[\mathrm{m}^{2}\right]$ \\
\hline B & buoyancy \\
\hline $\mathrm{C}$ & constant \\
\hline $\mathrm{C}_{\mathrm{p}}$ & specific heat $\left[\mathrm{J} \mathrm{kg}^{-1} \mathrm{~K}^{-1}\right]$ \\
\hline $\mathrm{F}_{\mathrm{b}}$ & buoyancy force $[\mathrm{N}]$ \\
\hline $\mathrm{g}$ & gravitational acceleration $\left[\mathrm{m} \mathrm{s}^{-2}\right]$ \\
\hline $\mathrm{g}^{*}$ & gravity level $\left[\mathrm{m} \mathrm{s}^{-2}\right]$ \\
\hline $\mathrm{H}$ & liquid layer height $[\mathrm{m}]$ \\
\hline $\mathrm{k}$ & thermal conductivity [ $\mathrm{W} \mathrm{m}{ }^{-1} \mathrm{~K}^{-1}$ ] \\
\hline $\mathrm{Ma}$ & Marangoni number \\
\hline $\mathrm{n}$ & unit normal vector \\
\hline $\mathrm{N}_{\mathrm{b}}$ & number of bubbles \\
\hline $\mathrm{p}$ & pressure $\left[\mathrm{N} \mathrm{m}^{-2}\right]$ \\
\hline $\operatorname{Pr}$ & Prandtl number \\
\hline $\mathrm{Q}_{\text {cond }}$ & heat transfer due to conduction [W] \\
\hline $\mathrm{Q}_{\mathrm{Ma}}$ & Marangoni heat transfer [W] \\
\hline $\mathrm{q}_{\text {cond }}^{\prime \prime}$ & heat flux due to conduction $\left[\mathrm{W} \mathrm{m}^{-2}\right.$ ] \\
\hline $\mathrm{q}_{\mathrm{Ma}}^{\prime \prime}$ & Marangoni heat flux $\left[\mathrm{W} \mathrm{m}^{-2}\right]$ \\
\hline $\mathrm{q}_{\mathrm{nc}}^{\prime \prime}$ & heat flux due to natural convection $\left[\mathrm{W} \mathrm{m}^{-2}\right]$ \\
\hline $\mathrm{q}_{\mathrm{st}}^{\prime \prime}$ & heat flux due to surface tension $\left[\mathrm{W} \mathrm{m}^{-2}\right]$ \\
\hline $\mathrm{q}_{\text {tot }}^{\prime \prime}$ & total heat flux $\left[\mathrm{W} \mathrm{m}^{-2}\right]$ \\
\hline $\mathrm{r}$ & radial direction $[\mathrm{m}]$ \\
\hline $\mathrm{Ra}$ & Rayleigh number \\
\hline $\operatorname{Re}$ & Reynolds number \\
\hline $\mathrm{R}_{\mathrm{b}}$ & bubble radius $[\mathrm{m}]$ \\
\hline $\mathrm{R}_{\mathrm{eff}, \mathrm{cw}}$ & effective radius, cold wall [m] \\
\hline $\mathrm{R}_{\mathrm{eff}, \mathrm{hw}}$ & effective radius, hot wall [m] \\
\hline $\mathrm{R}_{\text {enhancement }}$ & enhancement radius \\
\hline $\mathrm{R}_{\mathrm{Ma}}$ & Marangoni radius $[\mathrm{m}]$ \\
\hline $\mathrm{t}$ & time $[\mathrm{s}]$ \\
\hline $\mathrm{T}$ & temperature $[\mathrm{K}]$ \\
\hline $\mathrm{T}_{\mathrm{c}}$ & cold wall temperature $[\mathrm{K}]$ \\
\hline $\mathrm{T}_{\mathrm{h}}$ & hot wall temperature $[\mathrm{K}]$ \\
\hline $\mathrm{T}_{0}$ & reference temperature $[\mathrm{K}]$ \\
\hline $\mathrm{T}_{\mathrm{w}}$ & wall temperature $[\mathrm{K}]$ \\
\hline $\mathrm{v}$ & velocity $\left[\mathrm{m} \mathrm{s}^{-1}\right]$ \\
\hline $\mathrm{v}_{\mathrm{r}}$ & radial velocity $\left[\mathrm{m} \mathrm{s}^{-1}\right]$ \\
\hline $\mathrm{v}_{\mathrm{z}}$ & axial velocity $\left[\mathrm{m} \mathrm{s}^{-1}\right]$ \\
\hline $\mathrm{z}$ & axial direction $[\mathrm{m}]$ \\
\hline
\end{tabular}$$
A_{\text {eff,cw }} \quad \text { effective area, cold wall }\left[\mathrm{m}^{2}\right]
$$

\section{Greek Symbols}

$\begin{array}{ll}\alpha & \text { thermal diffusivity }\left[\mathrm{m}^{2} \mathrm{~s}^{-1}\right] \\ \beta & \text { expansion coefficient }\left[\mathrm{K}^{-1}\right] \\ \theta & \text { azimuthal direction }[\mathrm{rad}] \\ \mu & \text { dynamic viscosity }\left[\mathrm{kg} \mathrm{m}^{-1} \mathrm{~s}^{-1}\right] \\ \rho & \text { density }\left[\mathrm{kg} \mathrm{m}^{-3}\right] \\ \rho_{0} & \text { reference density }\left[\mathrm{kg} \mathrm{m}^{-3}\right] \\ \sigma & \text { surface tension }\left[\mathrm{N} \mathrm{m}^{-1}\right] \\ \tau & \text { shear stress }\left[\mathrm{N} \mathrm{m}^{-2}\right] \\ v & \text { kinematic viscosity }\left[\mathrm{m}^{2} \mathrm{~s}^{-1}\right]\end{array}$




\section{REFERENCES}

[1] Straub, J., Role of Surface Tension for Two-Phase Heat and Mass Transfer in the Absence of Gravity, Experimental Thermal and Fluid Science, vol. 9, pp. 253-273, 1994.

595 [2] Kao, Y. S., and Kenning, D. B. R., Thermocapillary Flow Near a Hemispherical Bubble on a Heated Wall, Journal of Fluid Mechanics, vol. 53, pp. 715-735, 1972.

[3] Larkin, B. K., Thermocapillary Flow Around Hemispherical Bubble, AIChE Journal, vol. 16, pp. 101-107, 1970.

600 [4] Kassemi, M., and Rashidnia, N., Bubble Dynamics on a Heated Surface, Proc. 3rd Microgravity Fluid Physics Conference, Cleveland, pp. 527-534, 1996.

[5] Marek, R., and Straub, J., The Origin of Thermocapillary Convection in Subcooled Nucleate Pool Boiling, International Journal of Heat and Mass Transfer, vol. 44, pp. 619-632, 2001.

[6] Reynard, C., Santini, R., and Tadrist, L., Experimental Study of Fluid-Wall Heat Transfer Induced by Thermocapillary Convection: Influence of the Prandtl Number, Comptes Rendus de l'Academie des Sciences Serie II b/Mecanique, vol. 331, pp. 237244, 2003.

610

[7] Arlabosse, P., Tadrist, L., Tadrist, H., and Pantaloni, J., Experimental Analysis of the Heat Transfer Induced by Thermocapillary Convection Around a Bubble, Transactions of the ASME. Journal of Heat Transfer, vol. 122, pp. 66-73, 2000.

615 [8] Wozniak, K., and Wozniak, G., Temperature Gradient Driven Flow Experiments of Two Interacting Bubbles on a Hot Wall, Heat and Mass Transfer/Waerme- und Stoffuebertragung, vol. 33, pp. 363-369, 1998.

[9] McGrew J. L., Bamford F. L., and Rehm T. R., Marangoni Flow: An Additional Mechanism in Boiling Heat Transfer, Science, vol. 153, pp. 1106-1107, 1966.

[10] Henry, C. D., Kim, J., and McQuillen, J., Dissolved gas effects on thermocapillary convection during boiling in reduced gravity environments, Heat and Mass Transfer, vol. 42, pp. 919-923, 2006.

[11] Straub, J., Zell, M., and Vogel, B., What we learn from boiling under microgravity, Microgravity Science and Technology, vol. 6, pp. 239-247, 1993.

[12] Raj, R., and Kim, J., Thermocapillary Convection During Subcooled Boiling in Reduced Gravity Environments, Proc. Interdisciplinary Transport Phenomena V: Fluid, Thermal, Biological, Materials and Space Sciences, Bansko, pp. 7.12-7.18, 2007.

[13] Lu, J. F., and Peng, X. F., Bubble Jet Flow Formation During Boiling of Subcooled Water on Fine Wires, International Journal of Heat and Mass Transfer, vol. 50, pp. 3966-3976, 2007.

[14] Wang, H., Peng, X. F., Christopher, D. M., Lin, W. K., and Pan, C., Investigation of Bubble-Top Jet Flow During Subcooled Boiling on Wires, International Journal of Heat and Fluid Flow, vol. 26, pp. 485-494, 2005.

640 [15] Barthes, M., Reynard, C., Santini, R., and Tadrist L., NonCondensable Gas Influence on the Marangoni Convection During a Single Vapour Bubble Growth in a Subcooled Liquid, Europhysics Letters, vol. 77, p. 5, 2007.

[16] Lee, H. S., and Merte, H., Jr., Pool Boiling Phenomena in MiKyongju, vol. 2, pp. 395-400, 1998.

[17] Kim, J., and Benton, J. F., Highly Subcooled Pool Boiling Heat Transfer at Various Gravity Levels, International Journal of Heat and Fluid Flow, vol. 23, pp. 497-508, 2002.

[18] Raake, D., Siekmann, J., and Chun, C. H., Temperature and Velocity Fields Due to Surface Tension Driven Flow, Experiments in Fluids, vol. 7, pp. 164-172, 1989.

[19] Petrovic, S., Robinson, T., and Judd, R. L., Marangoni Heat Transfer in Subcooled Nucleate Pool Boiling, International Journal of Heat and Mass Transfer, vol. 47, pp. 5115-5128, 2004.

[20] Reynard, C., Barthes, M., Santini, R., and Tadrist, L., Experimental Study of the Onset of the 3D Oscillatory Thermocapillary Convection Around a Single Air or Vapor Bubble: Influence on Heat Transfer, Experimental Thermal and Fluid Science, vol. 29, pp. 783-793, 2005.

[21] Wozniak, G., Optical Whole-Field Methods for ThermoConvective Flow Analysis in Microgravity, Measurement Science \& Technology, vol. 10, pp. 878-885, 1999.

[22] Wozniak, G., and Wozniak, K., Buoyancy and Thermocapillary Flow Analysis by the Combined Use of Liquid Crystals and PIV, Experiments in Fluids, vol. 17, pp. 141-146, 1994.

[23] Wozniak, K., Wozniak, G., and Roesgen, T., Particle-ImageVelocimetry Applied to Thermocapillary Convection, Experiments in Fluids, vol. 10, pp. 12-16, 1990.

[24] Arlabosse, P., Lock, N., Medale, M., and M., Jaeger, Numerical Investigation of Thermocapillary Flow Around a Bubble, Physics of Fluids, vol. 11, pp. 18-29, 1999.

[25] Reynard, C., Santini, R., and Tadrist, L., Experimental Study of the Gravity Influence on the Periodic Thermocapillary Convection Around a Bubble, Experiments in Fluids, vol. 31, pp. 440-446, 2001.

[26] O'Shaughnessy, S. M., and Robinson, A. J., Numerical Investigation of Bubble Induced Marangoni Convection, Annals of the New York Academy of Sciences (in press).

[27] Judd, R. L., and Hwang, K. S., A Comprehensive Model for Nucleate Pool Boiling Heat Transfer Including Microlayer Evaporation, Transactions of the ASME. Series C, Journal of Heat Transfer, vol. 98, pp. 623-629, 1976.

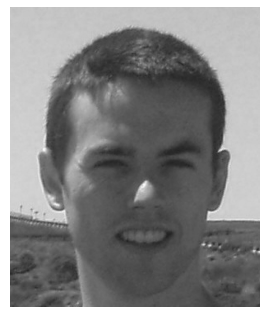

Séamus O'Shaughnessy is a Ph.D. student working as a member of the Fluids \& Heat Transfer Research Group in the Department of Mechanical \& Manufacturing Engineering, Trinity College, Dublin, Ireland. Currently he is working on a combined numerical and experimental study of thermocapillary convection in horizontal cavities caused by the presence of isolated bubbles maintained on a heated surface.

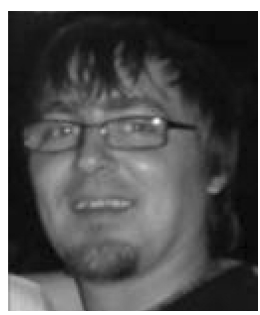

Anthony Robinson is a lecturer in mechanical engineering at Trinity College, Dublin, Ireland. He has extensive experience in both computational and experimental heat transfer and fluid dynamics. His areas of interest include pool boiling heat transfer, heat pipes, heat transfer and dry-out in capillary-porous media, thermocapillary convection, electrohydrodynamics (EHD), thermal interface materials, and jet impingement heat transfer. 